\title{
Automated Battery Swap and Recharge to Enable Persistent UAV Missions
}

\author{
Tuna Toksoz*, Joshua Redding ${ }^{\dagger}$, Matthew Michini ${ }^{\ddagger}$ \\ Bernard Michini ${ }^{\S}$, Jonathan P. How \\ Massachusetts Institute of Technology, Cambridge, MA 02139 \\ Matthew A. Vavrinall, John Vian** \\ Boeing Research \&s Technology, Seattle, WA 98108
}

\begin{abstract}
This paper introduces a hardware platform for automated battery changing and charging for multiple UAV agents. The automated station holds a buffer of 8 batteries in a novel dual-drum structure that enables a "hot" battery swap, thus allowing the vehicle to remain powered on throughout the battery changing process. Each drum consists of four battery bays, each of which is connected to a smartcharger for proper battery maintenance and charging. The hot-swap capability in combination with local recharging and a large 8-battery capacity allow this platform to refuel multiple UAVs for long-duration and persistent missions with minimal delays and no vehicle shutdowns. Experimental results from the RAVEN indoor flight test facility are presented that demonstrate the capability and robustness of the battery change/charge station in the context of a multi-agent, persistent mission where surveillance is continuously required over a specified region.
\end{abstract}

\section{Introduction}

As missions involving unmanned aerial vehicles (UAVs) lengthen in duration, frequent and robust refueling becomes critical to overall success. In the research setting, refueling typically means charging or swapping batteries onboard a small-scale UAV. Most recently, the use of a high energy laser beam to charge the battery during flight has been introduced. ${ }^{1}$ Automating and/or streamlining the recharging procedure has been the topic of much previous work. ${ }^{2-13}$ However, waiting for a battery to properly recharge can be very time-consuming, causing delays in the overall mission. Also, "cold" battery swapping techniques require a complete shutdown of the vehicle's onboard electronics as the spent battery is swapped for a new one. This adds further delay and a potential for losing onboard data and state information.

As slow recharge times and cold battery swaps are undesirable, this research introduces an automated, portable landing platform capable of "hot" swapping batteries such that the UAV remains powered up throughout the process. The automated station holds a buffer of seven batteries in a novel dual-drum structure that enables time-efficient swapping. Each drum consists of four battery

${ }^{*} \mathrm{~T}$. Toksoz is a SM Candidate in the Aerospace Controls Lab, MIT

${ }^{\dagger}$ J. Redding is a Ph.D. Candidate in the Aerospace Controls Lab, MIT

${ }^{\ddagger} \mathrm{M}$. Michini is an undergraduate Mechanical Engineer at McGill University

${ }^{\S}$ B. Michini is a Ph.D. Candidate in the Aerospace Controls Lab, MIT

` J. How is the R. C. Maclaurin Professor of Aeronautics and Astronautics, MIT and Associate Fellow of AIAA

"M. Vavrina is a Research Engineer at Boeing R\&T, Seattle, WA

** J. Vian is a Technical Fellow at Boeing R\&T, Seattle, WA 
bays, each of which can be connected to a charger for proper battery maintenance and charging. The hot-swap capability, in combination with local recharging and a large battery capacity allow this platform to refuel multiple UAVs for long-duration and persistent missions with minimal delays and without vehicle shutdowns.

Under a nominal payload (including a $1350 \mathrm{mAh} 12.6 \mathrm{~V}$ battery and USB camera), the quadrotors have a conservative flight time of about 7 minutes. The change/charge station can hold seven batteries, which enables a flight time of nearly an hour without utilizing the station's recharge capabilities. However, enabling the local recharge capability removes the upper limit on the operational flight time (when servicing a single vehicle), as a spent battery typically needs far less time to fully recharge than the flight time provided by the other batteries in the station (assuming that all seven batteries are fully charged initially). Experimental results, gathered from the RAVEN ${ }^{14,15}$ indoor flight test facility, are presented that demonstrate the capability and robustness of the battery change/charge station in the context of a multi-agent, persistent mission where surveillance is continuously required over a specified region. ${ }^{16}$

The remainder of the paper is organized as follows: The system hardware is described in detail in Section II followed by a discussion of the algorithms and software architecture in Section III. Section IV.B demonstrates the platorm's operation in a persistent search and tracking mission followed by the experimental results in Section IV.

\section{System Hardware}

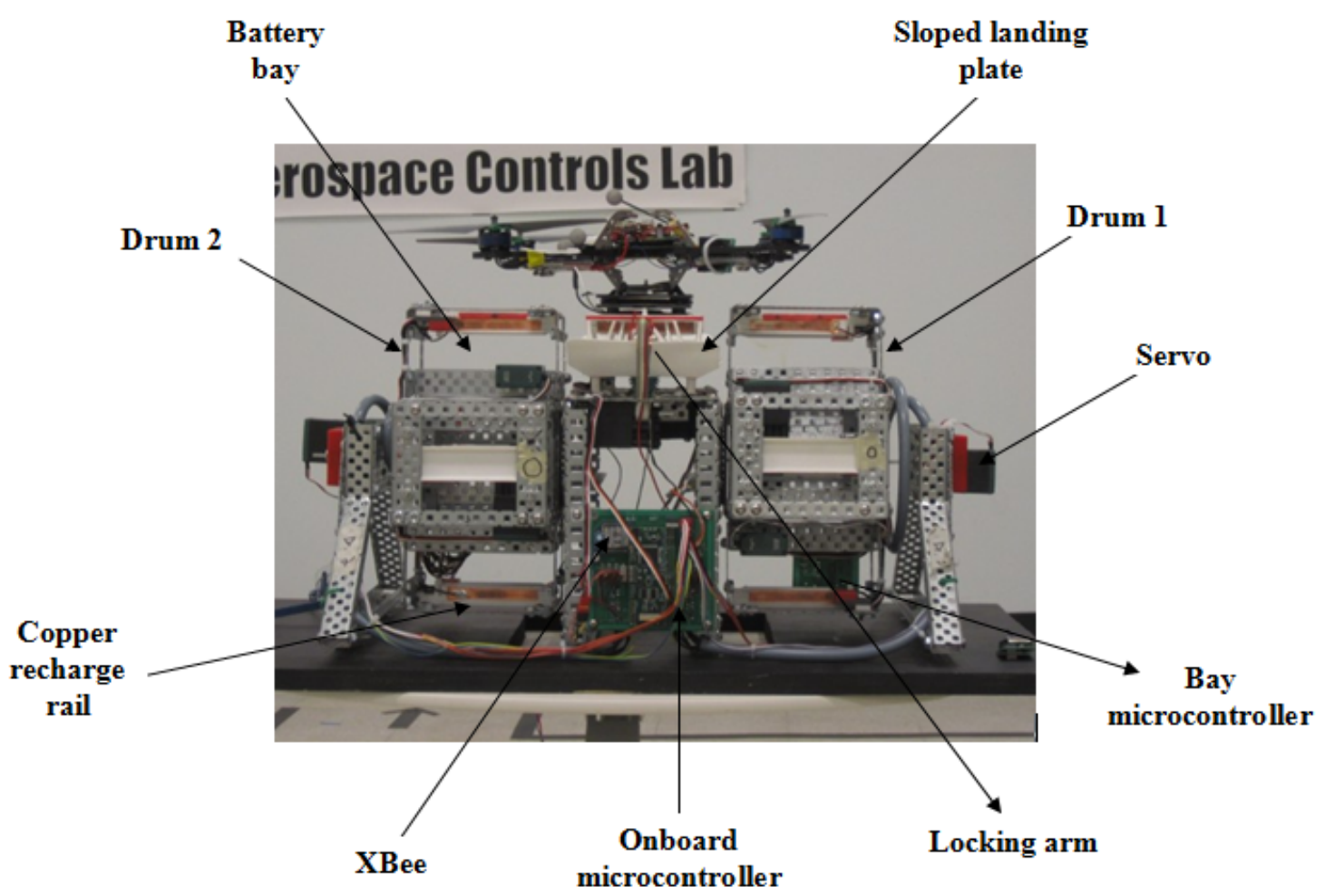

Fig. 1: The change/charge station is outfitted with 11 motors, 2 servos, 9 limit switch sensors, 2 rotational encoders, 3 custom PCBs, 3 microprocessors, an XBee wireless modem and optionally 8 battery chargers (not shown).

Figure 1 shows the complete system for automated battery maintenance. As seen, a retro-fitted quadrotor sits in a sloped landing plate and is held securely in place with two locking arms. The quadrotor is flanked by two rotating drums, each consisting of four battery bays which can carry (and optionally recharge) a single battery. The system as a whole provides a fully automated 


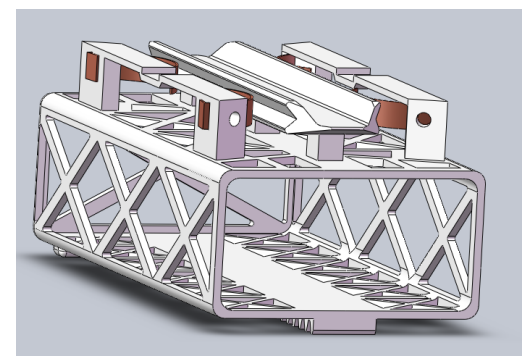

(a) CAD model of battery carriage

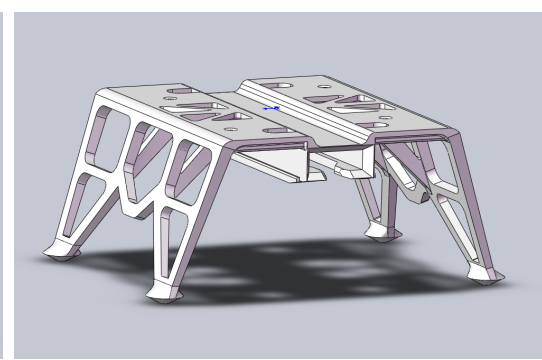

(b) CAD model of battery receiver

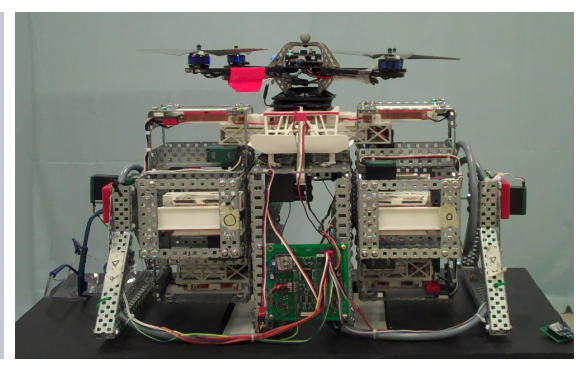

(c) Change/Charge station

Fig. 2: The three main hardware components of the developed battery management system: The battery carriage, the battery receiver and the station itself.

battery change/charge capability which can eliminate the need for a human operator to manually swap or recharge the batteries on the vehicle. Such a system offers an efficient and portable solution to the battery recharge problem.

Figure 2 shows the three main hardware components of the developed battery maintenance system: the battery carriage (Figure 2(a)), the battery receiver (Figure 2(b)) and the dual-drum change station (Figure 2(c)). The following sections discuss each of these components in more detail.

\section{II.A. Battery Carriage \& Receiver}

In order to enable the efficient changing of batteries, a special battery carriage has been designed, as shown in Figure 2(a). The carriage is a rectangular prism-shaped structure where the battery is secured inside. It provides power to the quadrotor through curved copper strips on either side of a T-shaped rail which ensures proper alignment as it slides into and out of recharge bays. A gear rack is built into the bottom of the carriage and allows corresponding pinion gears (found in the center and in each recharge bay of the change/charge station) to mesh with it, resulting in linear motion.

The T-shaped rail along the top of the carriage forms a sliding interface with the Tshaped channel built into the battery receiver, as shown in Figure 2(b). This provides a method for consistently aligning the carriage with the receivers on the quadrotor and in each bay. Figure 3 shows how one carriage slides out of a quadrotor receiver as another slides in. The combination of the curved copper strips and Trail provide a pressure-fit with the receiver that creates both a solid electrical connection and prevents the carriage from sliding out from underneath the quadrotor in mid-flight.

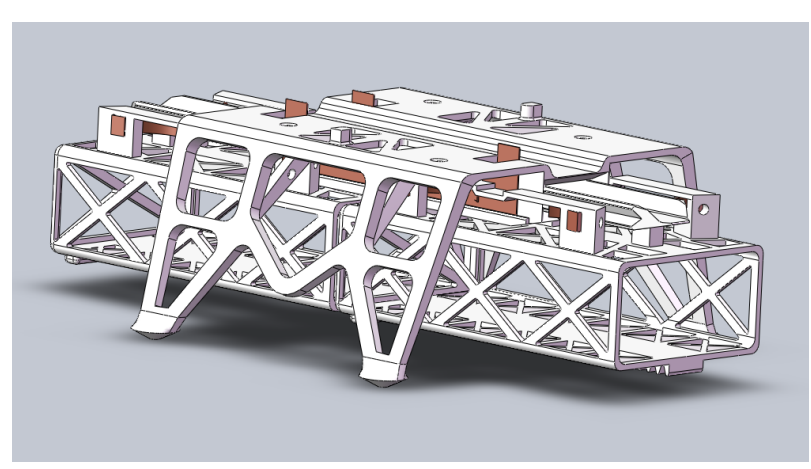

Fig. 3: Battery carriages sliding into and out of a quadrotor battery receiver

The quadrotor receiver is designed to be retro-fitted to any quadrotor helicopter while mating with the sloped landing plate on the change/charge station and accepting the battery carriage underneath. In such a design, the combined weight of the carriage and the receiver must be minimized so that the quadrotor can carry them without significantly shortening battery life. In order to meet this constraint, careful structural optimizations and material selections have been performed. The battery carriage and receiver designs were optimized using 3D modeling software and are created using a unique additive manufacturing process. 


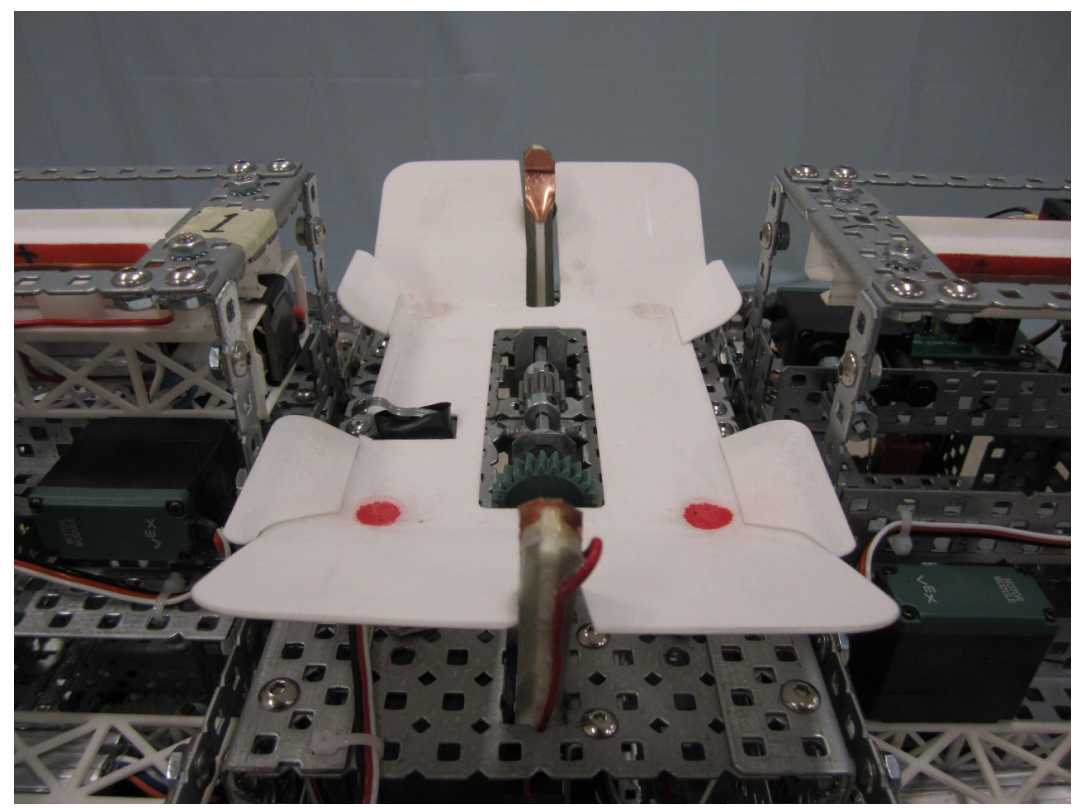

Fig. 4: Front view of the landing pad which contains sloped edges that slide the quadrotor into red spots. The central arms (front and back) help ensure vertical and horizontal alignment of the vehicle with the battery bays on the drums (left/right). The copper heads on the arms for transmitting power are clearly visible. The switch (left middle) detects that the vehicle/battery is in proper alignment. The center pinion gear that moves the battery carriage is also visible (middle center).

\section{II.B. Change/Charge Station}

Automatic battery changing/charging is accomplished by a combination of on-board microcontrollers and an off-board computer. The station utilizes a total of three microcontrollers: one on each drum to control the bay pinion motors, and one central unit that manages the internal and external communications and controls the center motor, locking arms, and both drum rotations.

Physically, the change/charge station consists of a center landing section situated between two opposing drums. The drums rotate about a common metal shaft to ensure proper concentric alignment. Encoders on each drum provide sub-degree rotational sensing and allow for precision control of the drum's alignment with the landing plate. The station's dual-drum structure enables simultaneous unloading of the spent battery and loading of the charged one in a single, linear motion, greatly simplifying the changing process.

Each drum consists of 4 battery bays, a PCB, and a PIC microcontroller. Each bay contains a motorized pinion gear (driven by the PIC) that can draw a battery carriage into, or push one out of, the bay itself. A switch, also mounted inside each bay, shuts the pinion motor off when the battery is fully inside. In addition, when a battery carriage is in a bay, electrical connections are made which enable recharging and the measurement of the battery's voltage level.

In the center section, a sloped landing plate, shown in Figure 4 assists in quadrotor alignment during landing. The four quadrotor feet conform exactly to four docking points (two of which are colored red in Figure 4) and thereby align the quadrotor to within $1 \mathrm{~mm}$ in $x, y$ and $z$. In addition, two separately-actuated semi-hooked arms, driven by servo motors, grasp the landed quadrotor and ensure its proper alignment, lock it securely to the station, and provide it with power as its battery is swapped. A small limit switch in the center section is activated when the battery carriage is properly aligned under the quadrotor. 


\section{Control Software}

Control of the change/charge station takes place both on- and off-board. The onboard firmware for the low-level control serves two functions: implementation of the motor control commands and broadcast sensor information. Commands such as to rotate a drum to a specific angle, or to engage a certain motor are sent from the off-board control software which makes the higher-level planning decisions. The sensor information, such as drum rotation angles, battery voltage levels and centerswitch status, is collected and sent to the off-board software at regular intervals and when the status of limit switch changes. This information is used to populate the state vector for the change station, denoted as

$$
s=\left[\begin{array}{llllll}
\theta_{1} & \theta_{2} & c & v_{1} & \cdots & v_{8}
\end{array}\right]^{T}
$$

where $\theta_{i} \in\left[0^{\circ}, 360^{\circ}\right)$ and indicates the rotation angle to the nearest degree of drum $i$. Further, $c \in 0,1$ denotes the status of the center-switch as either open or closed and $v_{i}$ the voltage level of the battery in bay $i$. The control software was designed to utilize this state information to make informed decisions regarding the presence of a battery in the center location, which battery to use next, and how to rotate the drums to accommodate the next swap. As illustrated in Figure 5, a typical battery changing sequence consists of the following steps:

1. A quadrotor lands on the center plate, turns off its motors, and sends a message to the off-board control software indicating that the landing is complete.

2. The off-board control software commands the change station to lock/align the quadrotor using its servo arms. A proper lock/alignment will activate the center switch, thereby notifying the off-board software.

3. With the quadrotor properly aligned and locked, the control software then scans the voltage levels of each bay and determines which bay is empty and which contains the battery with the highest voltage level. Commands are then sent to the change station to rotate the drums such that these two bays are aligned with the center section.

4. Once the drums are properly rotated, the control software sends commands to the change station to eject the charged battery from its bay while moving the spent battery out from under the quadrotor and toward the empty bay.

5. As the charged battery moves into its proper place in the receiver under the quadrotor, it activates the center switch and the center motor is stopped and the locking arms are released.

6. Upon activation of the center switch, the control software then sends a message to the quadrotor, clearing it for take-off. If this sequence is part of a larger mission, it is then up to the mission manager to decide when/if the quadrotor will actually take off.

\section{Experimental Results}

In this section, flight results are presented which show the average time required to swap a battery in a simplified, multi-swap test scenario. In addition, a persistent search and track mission is defined and flight results are given in the context of this more complex scenario. 


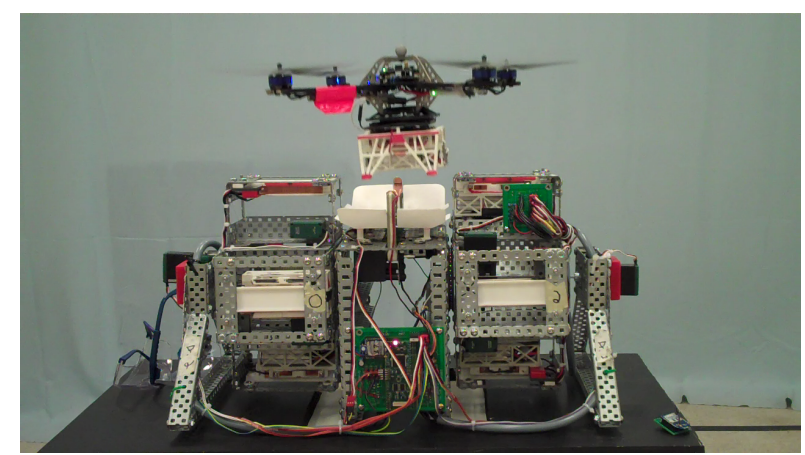

(a) Quadrotor hovers over pad and descends to land

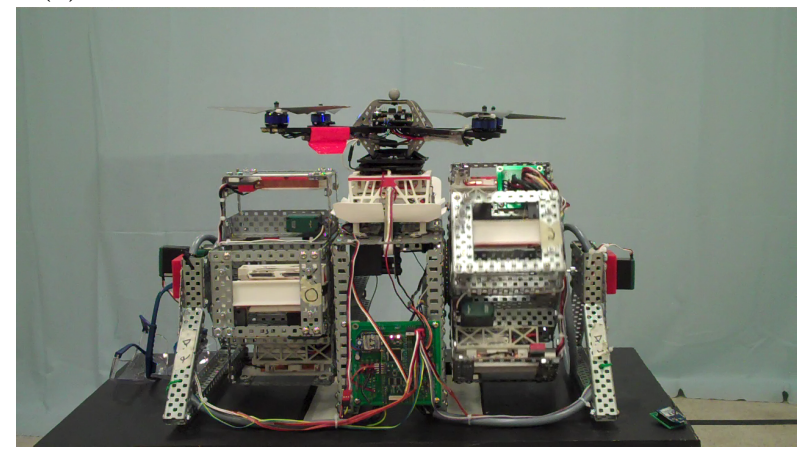

(c) New battery chosen and right drum rotates

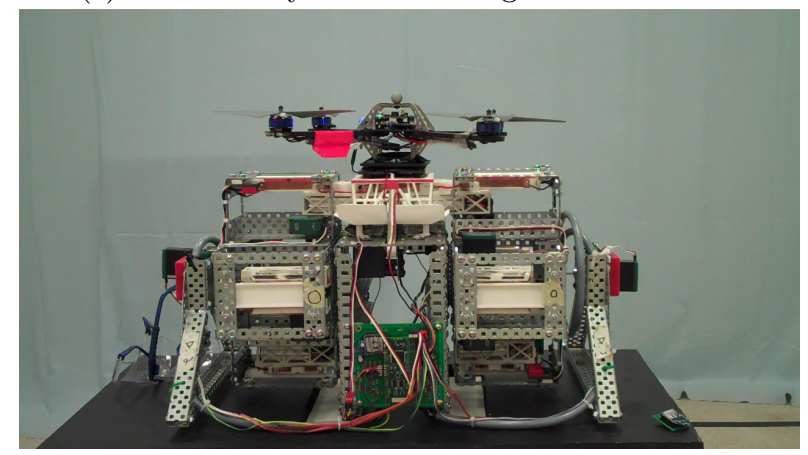

(e) Replacement battery pushed into place (right to left), moving the old battery into the left drum

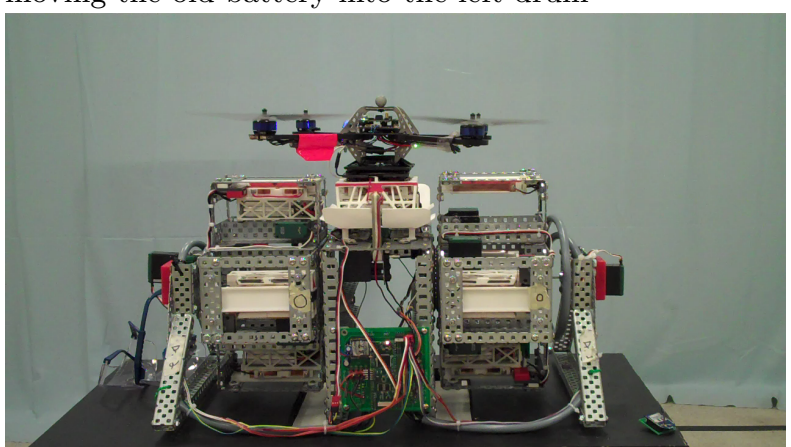

(g) Quadrotor motors restarted

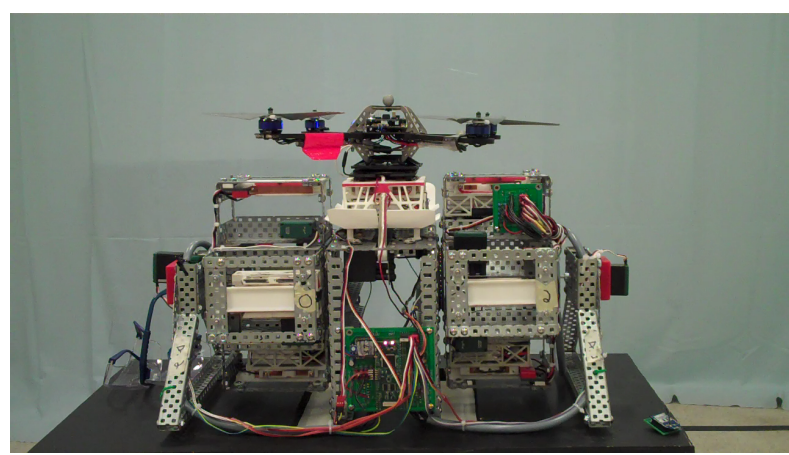

(b) Quadrotor clamped to pad with shore power

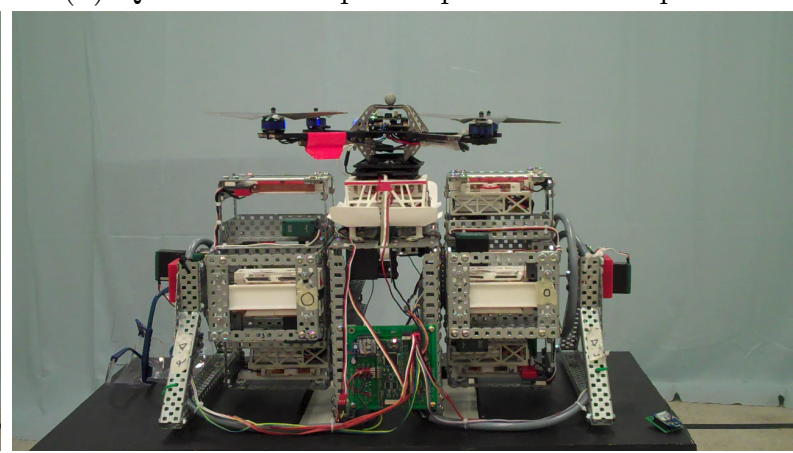

(d) Drums now aligned

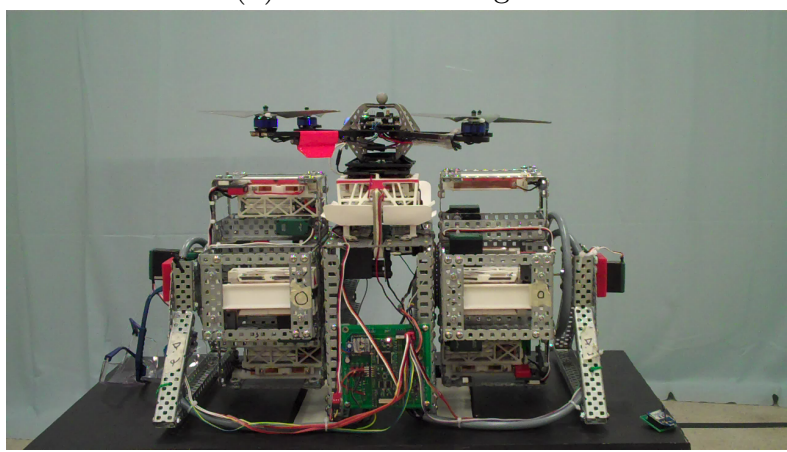

(f) After the battery swap is finished, the clamps are released

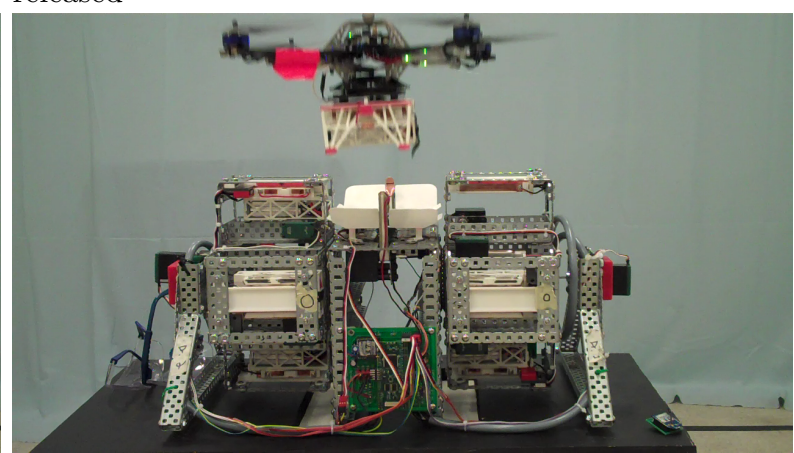

(h) Quadrotor takes off and returns to mission

Fig. 5: One battery swap sequence from a multi-swap mission. 


\section{IV.A. Time Required for Automated Battery Swaps}

To get an idea of the time required to swap a battery, a simple test was devised to perform $n$ swaps in quick succession, not waiting for the battery to drain between swaps. This allowed us to gather data corresponding to battery swaps only, without the added objectives of a mission to fulfill. During the test, a quadrotor was placed in the sloped landing plate and steps 2 through 5 of the sequence outlined in Section III were repeated - with the exception that in step 3, the drums were rotated according to a fixed, pre-determined ordering rather than by highest and lowest voltage bays.

Figure 6 shows a histogram of the elapsed time between steps 2 and 5 for 19 successive swaps. The mean and standard deviation of swap durations is measured to 11.8 seconds and 3.0 seconds respectively. The variation in swap times is likely due to differences in how far the

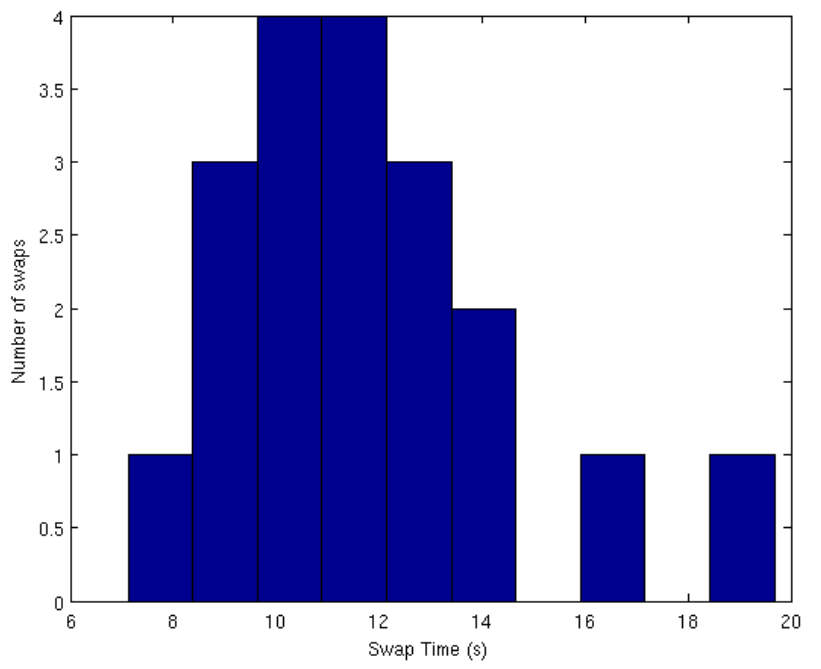

Fig. 6: Histogram showing the elapsed time between swap sequence steps 2 and 5 (as outlined in Section III) collected after 19 successive swaps. drum needed to rotate to align the correct battery.

\section{IV.B. Persistent Search and Track with Battery Recharge}

In the context of coordinating agents, many mission scenarios of interest are inherently longduration and require a high level of agent autonomy due to the high cost and complexity of human operation. The mission of interest in this paper is the problem of persistent search and track with communication constraints. ${ }^{10,16}$ In this problem, the mission area is divided into three regions labeled as the base, communication, and tasking areas.

The overall mission goal is to detect the target vehicles and perform persistent tracking and surveillance using ground and aerial vehicles, while satisfiying constraints including persistent existence of relay link in communication area. The quadrotors start the mission at the base area and travel to corresponding regions to perform search, track and communication duties, and return to the base for recharging or repair of failed actuators and sensors.

The communication area is the region between the base and tasking areas, and the communication constraint is such that a quadrotor must be in this area to successfully link the quadrotors in the tasking area with those at the base. This area also serves as the base for the two ground vehicles that can be tasked to track the targets when they are found.

The tasking area itself contains two or more target vehicles with several neutral vehicles. Upon arrival, the quadrotors search the tasking area and identify any vehicles found (via color-detection with a USB webcam). If they are classified as targets (rather than neutral), the ground vehicles are dispatched (if not already on site). The quadrotors continue to track the targets until the ground vehicles arrive, whereupon they return to searching the task area for other possible targets. Figure 7 provides snapshots of base, communication and tasking areas of the mission with 3 quadrotors, 2 ground vehicles, 2 targets and 2 neutral vehicles.

Several other constraints have to be satisfied during the mission. For example, each vehicle has a limited fuel capacity and thus the operational time is limited. If a vehicle runs out of fuel before it is recharged, then it will "crash" and can no longer be of use in the mission. In order to extend the duration of missions, the change/charge station described in II is placed in the base region, 


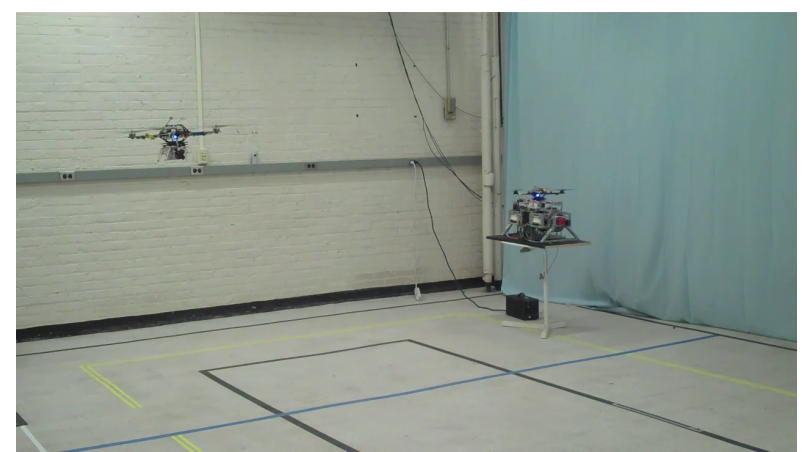

(a) Base and communication areas

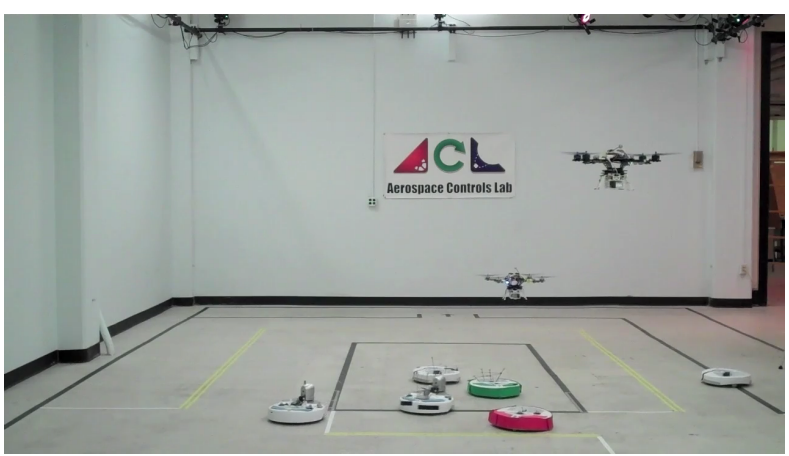

(b) Tasking area

Fig. 7: Snapshot from a typical mission. One quadrotor is at the charge/change station to refuel (back center of (a)), one quadrotor is at communication area to provide relay link (upper left of (a) and upper right of (b)), and the third is in the tasking area (middle center of (b)) tracking the green target vehicle while it waits for the ground vehicles (silver camera) to deploy.

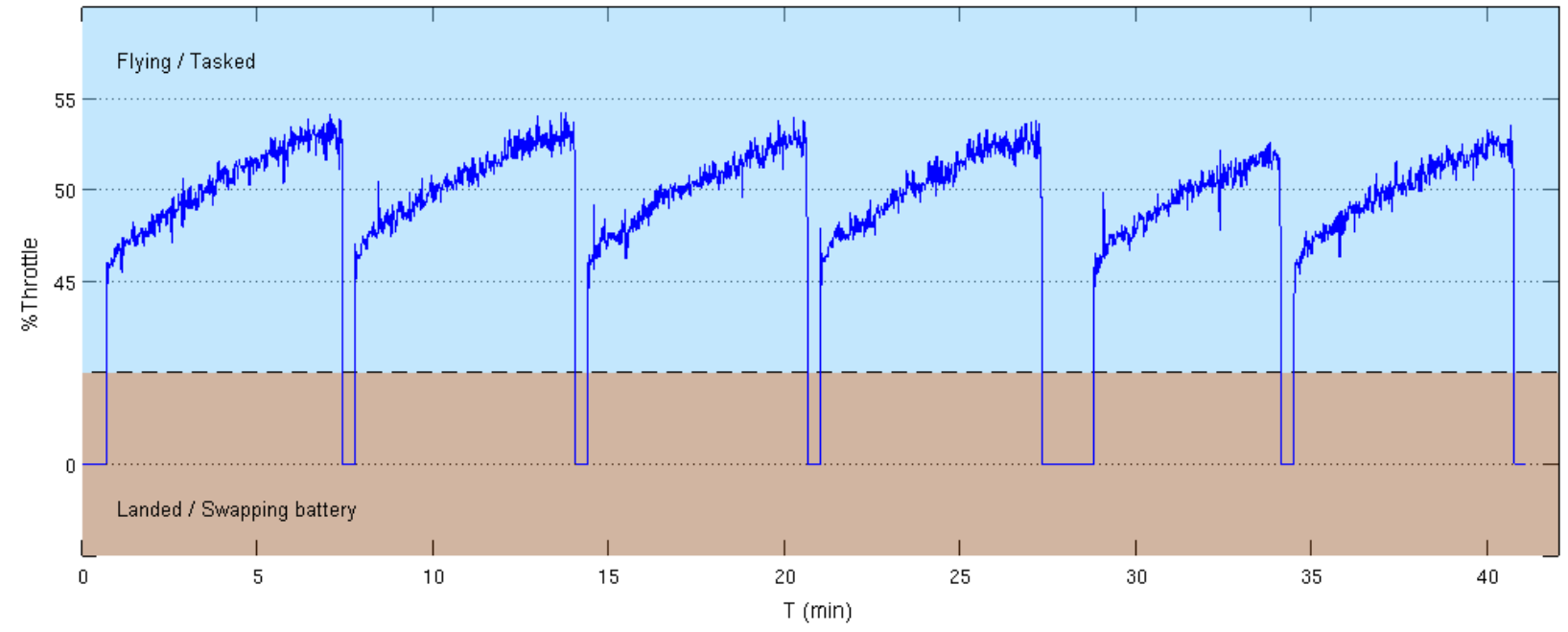

Fig. 8: Plot of the collective control input to a quadrotor helicopter during a $>40$ minute mission. As seen, battery swaps require less than 30 seconds between steps 1 and 6 of the swap sequence (described in Section III).

allowing quadrotors to autonomously refuel by changing their own batteries when they detect a low fuel level.

Each quadrotor in the mission can achieve a flight time of around seven minutes with a $1350 \mathrm{mAh}$ $12.6 \mathrm{~V}$ battery. The average charging time from a discharged state $(\approx 11.2 \mathrm{~V})$ is roughly 42 minutes. This indicates that a single change/charge station can provide an indefinite flight-time for single quadrotor. Our experiments use 3 quadrotors and 1 station, providing an upper-bound on mission time of 70 minutes.

Figure 8 shows the collective control input as it changes for a single quadrotor during an instance of the mission described above. This quadrotor swapped its battery a total of five times, with an average flight time of 6.18 minutes per battery. As shown, because of the integrator in the altitude control loop, the collective input level increases as the battery discharges from about $47 \%$ to $53 \%$ throttle. The sharp discontinuities in the plot represent the time spent sitting on the ground in the base area or on the change/charge station. Until $T=1$ minute, the quadrotor was on the ground in the base area, as is also the case for $T>41$ minutes. For the five cases in between, each 


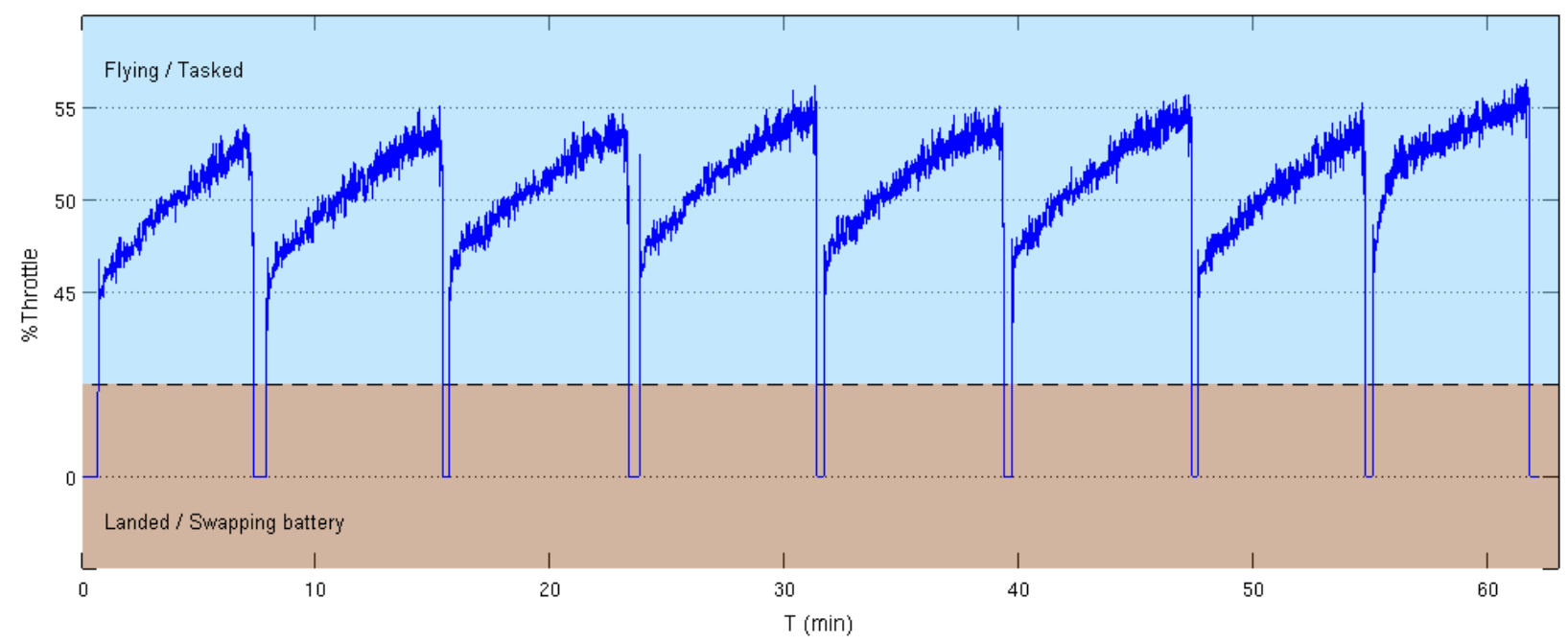

Fig. 9: Plot of the collective control input to a quadrotor helicopter during a second mission lasting $>60$ minutes. As seen, battery swaps require less than 30 seconds between steps 1 and 6 of the swap sequence (described in Section III).

represents a battery swapping instance. With one exception (at $T \approx 26$ minutes) the quadrotor was immediately called back out into the mission once the old battery was swapped out for a fresh one and the control software cleared the quadrotor for takeoff. For these swaps, the average swap time was 21.8 seconds, as measured from steps 1 through step 6 of the swap sequence outlined in Section III. This includes additional steps in comparison to the previous test, and the swap times are therefore slightly longer. In the case of the swap at $T \approx 26$ minutes, the quadrotor is not immediately called back out into the mission, and therefore waits on the change/charge station for almost 1.5 minutes. Overall, the change/charge station enables significantly extended mission times and improved efficiency resulting in only $7.8 \%$ down time.

Figure 9 also shows the collective control input as it changes for a single quadrotor during another instance of the mission described above. In this case, the quadrotor swapped its battery seven times for a mission time of over 60 minutes. Again, the change/charge station enabled a significantly extended mission time and resulted in only $4.2 \%$ down time.

\section{Conclusion}

This paper presented a hardware platform for autonomous battery changing and charging for quadrotor UAVs that are performing long-duration mission tests. The changer was designed to enable fast hot-swaps of batteries so that the vehicles can efficiently execute the mission (rather than spending extended periods of time at base). The changer was scaled to have eight battery bays, which should provide ample time to fully recharge the batteries before they are needed again. This enables fully autonomous long-term missions (multiple hours) for multiple vehicles and indefinite duration for a single vehicle. Future work will improve the onboard diagnostics of the quadrotors to keep track of the health state of the vehicles (including motor temperatures and battery voltage). 


\section{Acknowledgments}

This research was supported by Boeing Research \& Technology and in part by AFOSR (FA955009-1-0522). The views and conclusions contained herein are those of the authors and should not be interpreted as necessarily representing the official policies or endorsements, either expressed or implied, of the Air Force Office of Scientific Research or the U.S. Government.

\section{References}

[1] Nugent, T. and Kare, J., "Laser Power for UAVs," 2008, http://lasermotive.com/wpcontent/uploads/2010/04/Wireless-Power-for-UAVs-March2010.pdf.

[2] Hada, Y. and Yuta, S., "A First-Stage Experiment of Long Term Activity of Autonomous Mobile Robot - Result of Repetitive Base-Docking Over a Week," Experimental Robotics VII, ISER '00, Springer-Verlag, London, UK, 2001, pp. 229-238.

[3] Hada, Y. and Yuta, S., "A First-Stage Experiment of Long Term Activity of Autonomous Mobile Robot - Result of Respective Base-Docking Over a Week," Lecture Notes in Control and Information Sciences: Experimental Robotics VII, Vol. 271, 2001, pp. 229-238.

[4] Austin, D., Fletcher, L., and Zelinsky, A., "Mobile Robotics in the Long Term - Exploring the Fourth Dimension," Procedings of the IEEE/RSJ International Conference on Intelligent Robots and Systems, 2001.

[5] Kouzoubov, K. and Austin, D., "Autonomous Recharging for Mobile Robotics," Australian Conference on Robotics and Automation, Auckland, 2002, pp. 27-29.

[6] Silverman, M. C., Jung, B., Nies, D., and Sukhatme, G. S., "Staying alive longer: Autonomous robot recharging put to the test," Tech. Rep. CRES-03-015, Center for Robotics and Embedded Systems (CRES), University of Southern California, 2003, 2003.

[7] Vladimerouy, V., Stubbs, A., Rubel, J., Fulford, A., Strick, J., and Dullerud, G., "A hovercraft testbed for decentralized and cooperative control," American Control Conference (ACC), Boston, MA, July 2004, pp. 53325337.

[8] Cassinis, R., Bartolini, F. T. P., and Fedrigotti, R., "Docking and charging system for autonomous mobile robots," 2005, http://www.ing.unibs.it/ arl/docs/papers/05_008.pdf.

[9] De, P., Raniwala, A., Krishnan, R., Tatavarthi, K., Modi, J., Syed, N. A., Sharma, S., and Chiueh, T., "MiNT-m: An Autonomous Mobile Wireless Experimentation Platform," Proceedings of the 4th International Conference on Mobile Systems, Applications and Services, 2006.

[10] Valenti, M., Dale, D., How, J., and Vian, J., "Mission Health Management for 24/7 Persistent Surveillance Operations," Proceedings of the AIAA Guidance, Navigation, and Control Conference, Myrtle Beach, SC, August 2007.

[11] Dale, D. R., Automated Ground Maintenance and Health Management for Autonomous Unmanned Aerial Vehicles, Master's thesis, MIT Department of Electrical Engineering and Computer Science, June 2007.

[12] Valenti, M., Approximate Dynamic Programming with Applications in Multi-Agent Systems, PhD dissertation, Massachusetts Institute of Technology, Department of Electrical Engineering and Computer Science, June 2007.

[13] Swieringa, K., Hanson, C., Richardson, J., White, J., Hasan, Z., Qian, E., and Girard, A., "Autonomous battery swapping system for small-scale helicopters," IEEE International Conference on Robotics and Automation, May 2010, pp. 3335-3340.

[14] Valenti, M., Bethke, B., Fiore, G., How, J., and Feron, E., "Indoor Multi-Vehicle Flight Testbed for Fault Detection, Isolation, and Recovery," AIAA Guidance, Navigation, and Control Conference (GNC), Keystone, CO, August 2006 (AIAA-2006-6200).

[15] How, J. P., Bethke, B., Frank, A., Dale, D., and Vian, J., "Real-Time Indoor Autonomous Vehicle Test Environment," IEEE Control Systems Magazine, Vol. 28, No. 2, April 2008, pp. 51-64.

[16] Bethke, B., How, J., and Vian, J., "Multi-UAV Persistent Surveillance With Communication Constraints and Health Management," Proceedings of the AIAA Guidance, Navigation and Control Conference, Chicago, IL, August 2009 (AIAA-2009-5654). 